\title{
BMJ Open ASPIRE trial: study protocol for a double-blind randomised controlled trial of aspirin for overheating during exercise in multiple sclerosis
}

\author{
Anne Kever (D) , ${ }^{1}$ Katherine E Nelson, ${ }^{1,2,3}$ Ines M Aguerre, ${ }^{1,2,3}$ Claire S Riley, ${ }^{3}$ \\ Amelia Boehme, ${ }^{4}$ Nancy W Lee, ${ }^{5}$ Rebecca Strauss Farber, ${ }^{3}$ Seth N Levin, ${ }^{3}$ \\ Joel Stein, ${ }^{5}$ Victoria M Leavitt ${ }^{1,3}$
}

To cite: Kever A, Nelson KE, Aguerre IM, et al. ASPIRE trial: study protocol for a double-blind randomised controlled trial of aspirin for overheating during exercise in multiple sclerosis. BMJ Open 2020;10:e039691. doi:10.1136/ bmjopen-2020-039691

- Prepublication history and supplemental materials for this paper are available online. To view these files, please visit the journal online (http://dx.doi. org/10.1136/bmjopen-2020039691).

Received 22 April 2020 Revised 28 September 2020 Accepted 07 October 2020

Check for updates

(c) Author(s) (or their employer(s)) 2020. Re-use permitted under CC BY-NC. No commercial re-use. See rights and permissions. Published by BMJ.

For numbered affiliations see end of article.

Correspondence to Dr Victoria M Leavitt; VL2337@cumc.columbia.edu

\section{ABSTRACT}

Introduction The many benefits of exercise for persons with multiple sclerosis (MS) are well established, yet patients often refrain from exercise due to overheating and exhaustion. The present randomised controlled trial tests aspirin (acetylsalicylic acid (ASA)) as a convenient method to prevent overheating and improve exercise performance in persons with MS. The effects of ASA are compared with those of acetaminophen (APAP) and placebo.

Methods and analysis Participants are seen for a laboratory maximal exercise test on 3 separate days separated by at least 1 week. At each session, body temperature is measured before oral administration of a standard adult dose $(650 \mathrm{mg})$ of ASA, APAP or placebo. One hour after drug administration, participants perform a maximal ramp test on a cycle ergometer. Primary outcomes are (a) time to exhaustion (that is, time spent cycling to peak exertion) and (b) body temperature change. Crossover analyses will include tests for effects of treatment, period, treatment-period interaction (carryover effect) and sequence.

Ethics and dissemination Ethical approval was granted by the institutional review board at Columbia University Irving Medical Center (reference: AAAS2529). Results of the trial will be published in peer-reviewed scientific journals and presented at national and international conferences. Neurologists, physiatrists, primary care physicians and physiotherapists are important stakeholders and will be targeted during dissemination. Positive trial results have the potential to promote aspirin therapy, an inexpensive and readily available treatment, to reduce overheating and allow more persons with MS to benefit from exercise.

Trial registration number NCT03824938.

\section{INTRODUCTION}

Exercise holds numerous benefits for persons with multiple sclerosis (pwMS) ${ }^{12}$ including increased muscle strength, ${ }^{3}$ improved balance, ${ }^{4}$ decreased fatigue and depressive symptoms, ${ }^{5-7}$ and enhanced overall quality of life. ${ }^{8}$ Furthermore, a growing body of research supports exercise as potentially
Strengths and limitations of this study

- The rigorous double-blind, placebo-controlled, crossover design will expose all participants to three conditions, ensuring robust results and reducing the influence of confounding covariates.

- Positive findings from this trial will provide an effective, inexpensive, readily available, unobtrusive treatment that will avail more persons with multiple sclerosis (MS) the benefits of exercise.

- For this initial trial, our sample is limited to patients with relapsing-remitting MS although the potential benefits of aspirin should also be tested in persons with progressive forms of MS in future studies.

- The selected dose of aspirin (650 mg) was chosen based on two prior trials in MS as well as our own pilot trial, but future trials employing a lower dose are warranted.

disease modifying. In one study, patients with MS enrolled in a 24-week programme of exercise demonstrated increased serum levels of circulating brain-derived neurotrophic factor (critically involved in counteracting inflammatory processes of MS) ${ }^{9}$ and reduced proinflammatory circulating cytokines (often elevated during exacerbations of MS disease activity) relative to those who maintained a sedentary lifestyle over the same period of time. ${ }^{5}$ In addition, another study showed that just 12 weeks of aerobic exercise resulted in increased hippocampal volume and hippocampal functional connectivity, as well as improved memory in pwMS (compared with a stretching condition). ${ }^{10}$

Despite these and other well-documented benefits of exercise, individuals with MS sometimes avoid exercise due to transient discomforts of overheating and fatigue. ${ }^{11}$ Studies show that compared with their age-matched healthy peers, pwMS are less physically active 
regardless of disability and engage in less activity than their physical capacity allows. ${ }^{12-14}$ In fact, fewer than $20 \%$ of pwMS achieve adequate amounts of daily physical activity, levels that further decrease as the disease develops. ${ }^{15}$ The link between exercise and overheating has been known to the field for over a century: in 1890, Wilhelm Uhthoff's famous observation of the deleterious consequences of exercise for patients with MS precipitated a vast literature describing heat-related changes in MS. ${ }^{16} 17$

Exercise triggers the conversion of metabolic to mechanical energy, liberating approximately 30\%-70\% of the body's total energy as heat and thus causing an increase in core temperature. ${ }^{18}$ In everyone, as internal body temperature rises, physical fatigue increases and exercise performance worsens. ${ }^{19}$ In pwMS, slight increases in body temperature may lead to prohibitive levels of exhaustion during exercise. As a response to this, several cooling methods (eg, ice baths, cooling contraptions or garments, cooled environments) developed for pwMS have effectively reduced fatigue ${ }^{20}$ and improved exercise performance, ${ }^{21-25}$ although such methods can be cumbersome, prohibitively expensive or unavailable. Also, it is important to note that none of these prior trials of cooling methods in MS were conducted using doubleblind study design.

Our recent double-blind, crossover pilot trial demonstrated that aspirin (compared with placebo) significantly improved exercise performance and reduced exerciseinduced overheating in pwMS. ${ }^{26}$ Here, we present a detailed protocol of our large-scale trial of aspirin for exercise, ASPIRE: a double-blind, crossover randomised controlled trial (RCT) comparing the effects of aspirin (acetylsalicylic acid/ASA), acetaminophen (APAP) and placebo on exercise performance and exercise-induced body temperature increase in pwMS. The second arm, APAP, has been included for two reasons. First, if effective, APAP represents a potential alternative to ASA as a cooling treatment. Second, this third arm allows interrogation of mechanism: whereas aspirin is pleiotropic, APAP has much weaker/absent anti-inflammatory properties thereby isolating (to some degree) the antipyretic mechanism of ASA. ${ }^{27}$

Here, we test the hypothesis that ASA and APAP pretreatment (compared with placebo) will improve exercise performance and reduce exercise-induced body temperature increase during a controlled, laboratory maximal exercise test in pwMS.

\section{METHODS AND ANALYSIS \\ Study design}

This is a double-blind, crossover, placebo-controlled, randomised intervention design to examine the efficacy of an oral antipyretic (ie, ASA and APAP) to improve exercise performance and reduce exercise-induced body temperature in persons with MS. The trial comprises the following three arms: aspirin (ASA) was selected based on our prior exercise pilot as well as two prior trials showing its efficacy for reducing fatigue in non-exercising pwMS. ${ }^{28} 29$ ASA has a generally favourable safety profile, ${ }^{30}$ with no adverse events noted in these prior trials, nor more than minimal side effects (ie, no different than placebo). ${ }^{28}$ As noted, APAP was included to investigate mechanism: while both ASA and APAP are antipyretic, ASA has prominent anti-inflammatory properties. ${ }^{27}$ Furthermore, if APAP is found to be as effective as aspirin, it will provide another option that may be preferable for some pwMS. The third arm is placebo.

Participants are randomised to one of six sequences (see figure 1) with double-blind maintained by the study biostatistician $(\mathrm{AB})$ until data collection for the full sample is complete. Each participant engages in three exercise sessions held at the same time (within \pm 1 hour) on separate days with $\geq 1$ week intervals. All sessions are scheduled between 10:00 and 17:00 to reduce the influence of

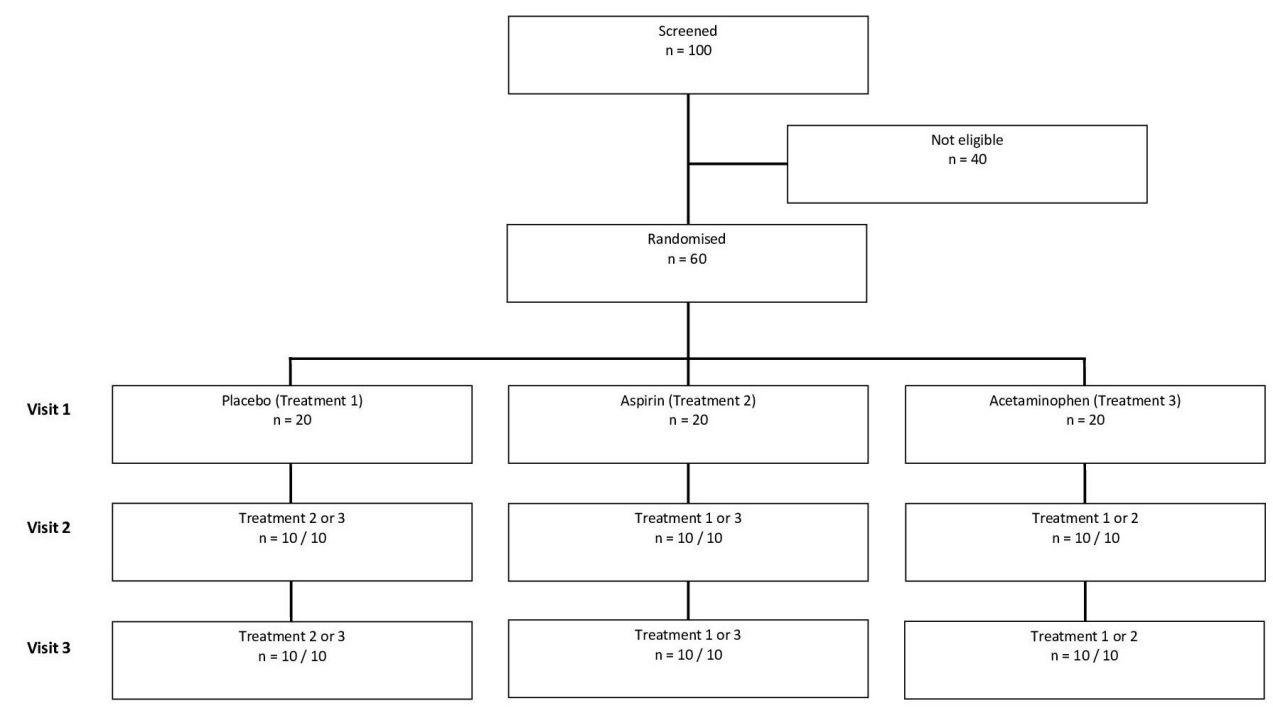

Figure 1 Design of the double-blind, crossover, randomised and placebo-controlled trial. 
circadian rhythm variability. Duration of each session is $\sim 2$ hours total (details in the Procedure section).

\section{Participants}

\section{Inclusion criteria}

Participants are included if they meet the following criteria: (1) diagnosis of relapsing-remitting MS (RRMS) based on the 2017 McDonald criteria, ${ }^{31}$ (2) aged between 18 and 65 years (upper limit is set to reduce effects of age-related activity level decreases and to ensure that our sample is representative of the population with relapseonset MS); (3) answer yes to the question: Is overheating during exercise a problem for you?; (4) low to moderate physical disability (ie, Expanded Disability Status Scale total score $\leq 6.0$ ); (5) relapse-free for 6 weeks prior; (6) body mass index $\leq 35$ (to reduce health-related confounds of obesity).

\section{Exclusion criteria}

Patients are excluded if they have any history of head injury, stroke or other neurological diseases/disorders; are currently taking antipyretics or pain medication daily; are currently in a depressive episode (screened with the Beck Depression Inventory-Fast Screen, excluded if total score $\geq 9)^{32}$ or present other psychiatric diagnoses; were formally diagnosed with sleep disorder, pulmonary disease, heart disease or other heart problems; suffer from vascular disease of the legs, high blood pressure (BP); currently take medications for high BP or any heart problem; have diabetes mellitus or problems with blood sugar levels; present any contraindications to ASA use, that is, history of confirmed peptic ulcer, gastrointestinal or severe gynaecological bleeding; have tarry stool or faecal occult blood, syndrome of asthma, rhinitis or nasal polyps; present any contraindications to APAP use, that is, severe active hepatic disease, hepatitis $\mathrm{C}$ virus.

\section{Sample size determination}

In a pilot trial with 12 participants, effect sizes for both outcomes (time to exhaustion/TTE and body temperature) were very large (Cohen's $d=1.45)$. Here, we conservatively estimated medium-sized effects $(0.5)$ to calculate a power estimate for the proposed trial. With significance set at $\alpha=0.05$ for two-tailed paired t-test, a three-arm crossover sample of 54 will yield 0.95 power to detect differences between interventions. Our plan to enrol 60 participants allows for $10 \%$ attrition, which accounts for both participant drop-out as well as exclusion due to relapses.

\section{Recruitment and consent}

Based on a priori power analysis, 60 patients with a diagnosis of RRMS will be enrolled. The sample is projected to comprise $66 \%$ women and $33 \%$ men, consistent with disease demographics. All participants will be recruited from the Multiple Sclerosis Center at the Columbia University Irving Medical Center (CUIMC). One hundred patients will be screened, conservatively estimating that $60 \%$ will meet eligibility based on our pilot trial. ${ }^{33}$ The medical director of the MS centre (CSR) and a dedicated research coordinator (KEN) lead patient recruitment and confirm the disease-specific inclusion criteria. To avoid coercion, participants are not invited to participate by their treating neurologist. Instead, a research coordinator provides participants with an institutional review board (IRB)-approved recruitment advertisement for the study, with instructions to contact the research coordinator if she/he is interested in participating. Participants receive weekly reminder emails and phone calls to promote patient retention and completion of follow-up visits. Data from participants who discontinue or deviate from intervention protocols are excluded from final analyses but will be retained for inspection of any systematic differences. Participants are expressly informed that participation in this study is voluntary, not part of their medical care, affords no advantage to their health and that refusal to participate results in no negative consequences. Before commencement of the study, participants sign a consent form approved by the IRB at CUIMC (for a model, please see online supplemental materials). The latter also details the provisions taken in case of injury or harm as a result of participating in the study, that is, care provided through CUIMC or any other health provider. Participants receive $\$ 100$ at the completion of their third exercise session as compensation for time and travel expenses.

\section{Allocation, randomisation, blinding and data protection}

Using a random number generator, each participant is assigned to one of six randomisation schedules: placeboAPAP-ASA, placebo-ASA-APAP, ASA-APAP-placebo, ASAplacebo-APAP, APAP-ASA-placebo, APAP-placebo-ASA. The research pharmacy (providing all study drug) receives a randomisation schedule to ensure that necessary procedures for maintaining the double-blind are in place. The key containing participant ID and randomisation schedule assignment is kept by the study biostatistician for the duration of the active period of the trial and will be provided to the study's principal investigator only after all participants have been tested. To test effectiveness of blinding, participants are asked via paper questionnaire which condition they think they were in at the end of each session. In order to safeguard confidentiality, participants are identified by a number and the master key is password protected, and accessible only to personnel directly involved in the study.

\section{Intervention and procedure}

Pharmaceutical pretreatment

Room temperature is held constant to within $\pm 5^{\circ} \mathrm{F}$ and recorded at the start of each session. Participants are instructed to not eat 2 hours prior to their session (to avoid subsequent increases in body temperature) ${ }^{34}$ and to refrain from use of ASA or APAP within 24hours of their study visit. On arrival to the lab, written informed consent is obtained from all participants. Body temperature is measured with a tympanic thermometer (right ear; Braun Thermoscan IRT), a standard, non-invasive 


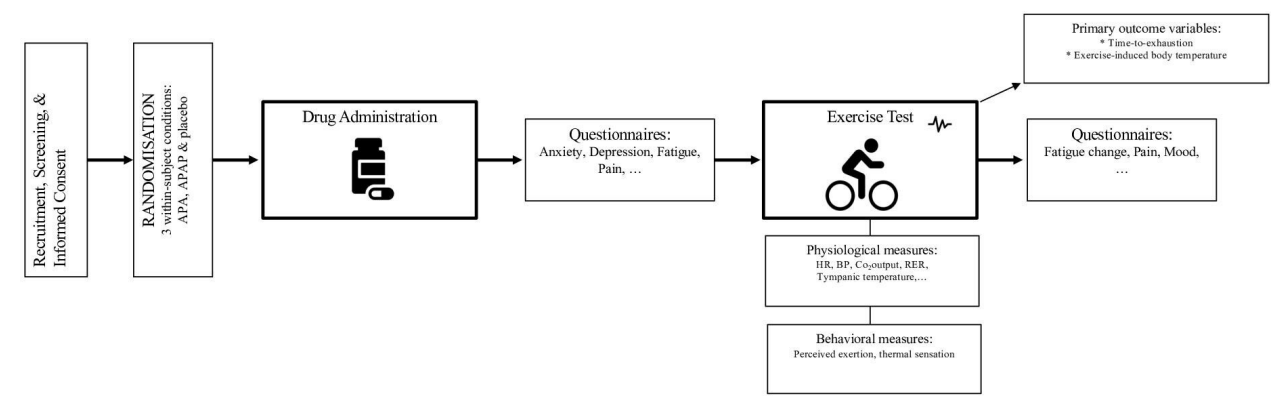

Figure 2 Overview of experimental procedure. APA, acetylsalicylic acid; APAP, acetaminophen; BP, blood pressure; HR, heart rate; RER, respiratory exchange ratio.

method which best approximates core body temperature. $^{35} 36$ Participants receive one of three treatments (ASA, APAP, placebo) at each session prior to the exercise test. Order of treatment is randomised and counterbalanced, with each participant serving as his/her own control (see figure 2). Treatment is administered as one capsule containing $650 \mathrm{mg}$ (ie, standard adult dose) of ASA, APAP or placebo. ASA dose was selected on the basis of prior successful RCTs of ASA to reduce fatigue in non-exercising patients with MS. ${ }^{28}$ Overencapsulation of ASA and manufacture of placebo is handled by the CUIMC research pharmacy. Time of drug administration is recorded, and exercise does not begin until 1 hour later to allow for peak serum level to be reached. ${ }^{37}$ During this time, participants complete a series of questionnaires to characterise the sample (for details, see the Behavioural measures section).

\section{Exercise test}

Exercise tests are conducted by a certified exercise physiologist (NWL). All procedures are consistent with American College of Sports Medicine guidelines. ${ }^{38}$ All exercise testing equipment is current and certified for use with human subjects. Cardiopulmonary exercise testing is performed using Vmax Encore Metabolic Cart (CareFusion Corp, San Diego, California, USA) and ECG monitoring is done with a 12-lead system attached to the Vmax Encore Metabolic Cart with cardiosoft software. Peak ventilatory capacity (maximum voluntary ventilation) is determined before the exercise test via Vmax Encore System (CareFusion Corp). Maximum aerobic fitness (VO2max) is measured during the graded maximal exercise test on a VIAsprint 150P electronic-braked cycle ergometer (CareFusion Corp).

Participants complete a 5-minute resting phase followed by a 3-minute warm-up and then start the progressive ramped exercise. An individualised ramping protocol is determined by a certified exercise physiologist based on the exercise frequency and intensity that each patient self-reports. For participants who exercise irregularly and maximum zero to two times/week, resistance increases by $5 \mathrm{~W} / \mathrm{min}$, those who exercise regularly two to four times/week follow a $10 \mathrm{~W} / \mathrm{min}$ protocol, while those who exercise more than four times/ week are assigned to a $15 \mathrm{~W} / \mathrm{min}$ protocol. However, depending on the type of exercise, ramping protocols can be adapted (eg, four yoga sessions/week vs four high-intensity cycling sessions/week). Participants are instructed to maintain a cadence of 50-60 revolutions/ min (RPM) for as long as possible. The test is terminated when cadence drops below 40 RPM for $\geq 5 \mathrm{~s}$, or when the participant reaches volitional exhaustion in accordance with the American Thoracic Society standard test termination criteria. ${ }^{39}$

The following biophysiological data are collected during the exercise session (to ensure participant safety ${ }^{38}$ and for exploratory analyses): heart rate (HR) (every $60 \mathrm{~s}$ ), BP and tympanic temperature (every 120 s from 2 min onwards), respiratory exchange ratio, minute ventilation (ie, total sum of volume delivered over a minute), expired oxygen, carbon dioxide output and maximum watts unleashed during the test. At exercise termination, total TTE (that is, time before the given work rate cannot be maintained and there is cessation of the exercise), our main outcome variable, is recorded.

\section{Behavioural measures}

\section{Pre-exercise}

During the 1-hour period between drug administration and start of the exercise test, participants complete the following questionnaires: the 14-item Hospital Anxiety and Depression Scale (frequency score from 0 to 3$)^{40}$ and the 9-item Fatigue Severity Scale (agreement score from 1 to 7$)^{41}$ to characterise the sample; the Paffenbarger Physical Activity Scale ${ }^{42}$ for post-hoc evaluation of physical activity levels, the day of last menstrual cycle (if women), number of hours slept during prior night, any physical/outdoor activities engaged in today, presence of illness in self or family members today; and 10-point visual analogue scales (VAS) of pain, fatigue and sadness. These variables are collected to enable future exploratory analyses.

\section{During exercise}

The Borg Ratings of Perceived Exertion Scale (RPE) for breathing (RPEbr) and muscle fatigue (RPEleg) ${ }^{43} 44$ and the Thermal Sensation Scale (ASHRAE Scale) ${ }^{45}$ are completed at $60 \mathrm{~s}$ intervals throughout the session (consistent with Collett et al). ${ }^{46}$ 


\section{Post-exercise}

At termination, participants provide a last RPEbr and RPEleg, complete a 10-point VAS of fatigue, mood and pain, and rate their Global Fatigue Change. ${ }^{28}$

\section{Trial status}

Participant recruitment commenced on 20th of February 2019, and data collection completion is anticipated for February 2021. Currently, 34 participants have been enrolled in the study, and 16 have completed all sessions. Data collected until now will not be reported in this paper. The diversity of our sample is notable: $26 \%$ men; race/ ethnicity: 9\% Black, 6\% Hispanic/Latinx. Recruitment has been facilitated by the appeal of this study to individuals with MS, for whom the idea of a possible treatment to reduce the discomfort of overheating during exercise resonates. Participants in the trial are uniquely motivated on completing a session; one patient recently stated: "I always feel so much better after these study visits!”

\section{Data analysis}

Statistical methods recommended for the proper analysis of crossover trial data will be used (for details, see Wellek and Blettner). ${ }^{47}$ Our primary outcomes evaluate differences between treatment condition (ASA, APAP and placebo) with respect to (a) TTE (that is, length of time spent exercising) and (b) increase in exercise-induced body temperature $\left({ }^{\circ} \mathrm{F}\right)$.

Differences between pretreatment conditions (ASA, APAP, placebo) will be analysed separately by sequence group to account for possible order effects (ie, stepwise assessment of outcome differences across six possible sequences). Although significant differences in outcome variables between ASA and APAP are not anticipated, we are interested in seeing how they compare in the full analyses. A mixed-effects linear model will be used to account for repeated measurements of each outcome, period effect, sequence effect and carryover effect. Using mixedeffects linear model will enable us to model differences in outcomes both within and between patients (intrapatient and interpatient variability). If assumptions for mixedeffects linear model are not met and log transformation is not appropriate, non-linear mixed-effects models will be used. Moreover, exploratory analyses will examine potential pretreatment differences for additional outcome measures, such as physiological indices (eg, HR, BP) and behavioural self-reports (eg, RPEs, fatigue, pain).

\section{Patient and public involvement}

The ASPIRE trial was developed through a commitment to clinically informed and therapeutically meaningful translational research, building on more than 125 years of observational evidence for the detrimental effects of exercise-induced overheating in MS, coupled with anecdotal patient reports of overheating as a deterrent to exercise. Our centre's patients with MS have been involved throughout the development and execution of the study, providing valuable feedback to inform our selection of outcome variables and optimise our study design. Moreover, participant feedback from a previously conducted small pilot trial $^{26}$ was considered while developing the present study.

\section{ETHICS AND DISSEMINATION}

This study has been approved by the CUIMC IRB (reference: AAAS2529) and all participants will provide informed consent prior to the study commencement. Results will be disseminated at relevant national and international meetings, conferences and workshops. We also plan to publish our findings in peer-reviewed, high-impact journals and aim to encourage other research groups to investigate clinically relevant questions using rigorous experimental designs (see eg, Dalgas et al for a cogent discussion on how to bolster the quality and scope of exercise studies in MS). ${ }^{48}$ Positive results will also be shared with medical caregivers, especially MS clinical practitioners. Furthermore, our MS centre at CUIMC distributes a biannual newsletter for patients that reports current research opportunities and highlights published study results to create a $360^{\circ}$ feedback loop and acknowledge the essential role of patients in the success of our research. Patient feedback reveals this to be highly motivating.

\section{DISCUSSION}

Overall, the present study attempts to test the effectiveness of aspirin as a treatment to reduce overheating (and associated discomfort) during exercise in pwMS. Several cooling methods already exist but they are cost prohibitive in many instances, unavailable or cumbersome. Exercising in an air-conditioned gym, for instance, appears at first glance to be a simple and obvious recommendation. But not everyone has the financial and practical means to regularly visit a gym. Aspirin, however, is inexpensive, Food and Drug Administration approved, readily available and has a favourable safety profile. ${ }^{30}$ If this trial is successful and confirms the finding of our pilot study, ${ }^{33}$ it will support the first widely available symptomatic treatment to facilitate exercise in MS with the potential to make a meaningful difference. For research on exercise in MS, having a pretreatment to reduce exercise-induced overheating and exhaustion will mitigate sample bias, that is, we can speculate that the extant literature may be biased toward study samples that over-represent patients for whom overheating is not an issue, therefore limiting generalisability. Furthermore, a positive outcome of the ASPIRE trial would set the stage for future work investigating daily aspirin use to increase free living physical activity levels or feasibility of aspirin pretreatment for individuals with progressive forms of MS.

\section{Author affiliations}

${ }^{1}$ Translational Cognitive Neuroscience Laboratory, Department of Neurology, Columbia University Irving Medical Center, New York, NY, USA

${ }^{2}$ Center for Translational \& Computational Neuroimmunology, Department of Neurology, Columbia University Irving Medical Center, New York, NY, USA 
${ }^{3}$ Multiple Sclerosis Center, Department of Neurology, Columbia University Irving Medical Center, New York, NY, USA

${ }^{4}$ Department of Neurology and Epidemiology, Columbia University Irving Medical Center, New York, NY, USA

${ }^{5}$ Department of Rehabilitation and Regenerative Medicine, Columbia University Vangelos College of Physicians and Surgeons, New York, NY, USA

Contributors AK and VML wrote the manuscript and made alterations based on coauthors' comments. KEN coordinated patient recruitment and data collection, and provided important input during manuscript drafting. IMA coordinated patient recruitment and reviewed the manuscript for intellectual content. CSR reviewed the manuscript for intellectual content. NWL conceptualised the exercise test and informed the choice of the physiological outcome measures. AB provided the statistical analysis plan. SNL and RSF helped with patient recruitment. JS reviewed the manuscript for intellectual content. VML, as the principal investigator, designed and conceptualised the study, and has overall leadership of the trial. All authors reviewed draft versions and approved the final manuscript.

Funding This work was supported by a grant from the National Institutes of Health (National Center for Medical Rehabilitation Research within the National Institute of Child Health and Human Development) to VML (HD091836-01A1).

Competing interests None declared.

Patient consent for publication Not required.

Provenance and peer review Not commissioned; externally peer reviewed.

Supplemental material This content has been supplied by the author(s). It has not been vetted by BMJ Publishing Group Limited (BMJ) and may not have been peer-reviewed. Any opinions or recommendations discussed are solely those of the author(s) and are not endorsed by BMJ. BMJ disclaims all liability and responsibility arising from any reliance placed on the content. Where the content includes any translated material, BMJ does not warrant the accuracy and reliability of the translations (including but not limited to local regulations, clinical guidelines, terminology, drug names and drug dosages), and is not responsible for any error and/or omissions arising from translation and adaptation or otherwise.

Open access This is an open access article distributed in accordance with the Creative Commons Attribution Non Commercial (CC BY-NC 4.0) license, which permits others to distribute, remix, adapt, build upon this work non-commercially, and license their derivative works on different terms, provided the original work is properly cited, appropriate credit is given, any changes made indicated, and the use is non-commercial. See: http://creativecommons.org/licenses/by-nc/4.0/.

ORCID iD

Anne Kever http://orcid.org/0000-0002-3860-6839

\section{REFERENCES}

1 Owen N, Sparling PB, Healy GN, et al. Sedentary behavior: emerging evidence for a new health risk. Mayo Clin Proc 2010;85:1138-41.

2 Veldhuijzen van Zanten JJCS, Pilutti LA, Duda JL, et al. Sedentary behaviour in people with multiple sclerosis: is it time to stand up against MS? Mult Scler 2016;22:1250-6.

3 Petajan JH, Gappmaier E, White AT, et al. Impact of aerobic training on fitness and quality of life in multiple sclerosis. Ann Neurol 1996;39:432-41.

4 Gunn H, Markevics S, Haas B, et al. Systematic review: the effectiveness of interventions to reduce falls and improve balance in adults with multiple sclerosis. Arch Phys Med Rehabil 2015;96:1898-912.

5 Alvarenga-Filho H, Sacramento PM, Ferreira TB, et al. Combined exercise training reduces fatigue and modulates the cytokine profile of T-cells from multiple sclerosis patients in response to neuromediators. J Neuroimmunol 2016;293:91-9.

6 Cooney GM, Dwan K, Greig CA, et al. Exercise for depression. Cochrane Database Syst Rev 2013;9:CD004366.

7 Pilutti LA, Greenlee TA, Motl RW, et al. Effects of exercise training on fatigue in multiple sclerosis. Psychosom Med 2013;75:575-80.

8 Motl RW. Benefits, safety, and prescription of exercise in persons with multiple sclerosis. Expert Rev Neurother 2014;14:1429-36.

9 Wens I, Keytsman C, Deckx N, et al. Brain derived neurotrophic factor in multiple sclerosis: effect of 24 weeks endurance and resistance training. Eur J Neurol 2016;23:1028-35.

10 Leavitt VM, Cirnigliaro C, Cohen A, et al. Aerobic exercise increases hippocampal volume and improves memory in multiple sclerosis: preliminary findings. Neurocase 2014;20:695-7.
11 Christensen ME, Brincks J, Schnieber A, et al. The intention to exercise and the execution of exercise among persons with multiple sclerosis - a qualitative metasynthesis. Disabil Rehabil 2016;38:1023-33.

12 Marrie RA, Horwitz R, Cutter G, et al. High frequency of adverse health behaviors in multiple sclerosis. Mult Scler 2009;15:105-13.

13 Casey B, Coote S, Galvin R, et al. Objective physical activity levels in people with multiple sclerosis: meta-analysis. Scand J Med Sci Sports 2018;28:1960-9.

14 Motl RW, McAuley E, Snook EM. Physical activity and multiple sclerosis: a meta-analysis. Mult Scler 2005;11:459-63.

15 Motl RW, McAuley E, Sandroff BM. Longitudinal change in physical activity and its correlates in relapsing-remitting multiple sclerosis. Phys Ther 2013;93:1037-48.

16 Opara JA, Brola W, Wylegala AA, et al. Uhthoff's phenomenon 125 years later - what do we know today? J Med Life 2016;9:101-5.

17 Panginikkod S, Rukmangadachar L. Uhthoff phenomenon. StatPearls Publishing, 2019.

18 Edwards $\mathrm{RH}$, Hill DK, Jones DA. Heat production and chemical changes during isometric contractions of the human quadriceps muscle. J Physiol 1975;251:303-15.

19 González-Alonso J, Teller C, Andersen SL, et al. Influence of body temperature on the development of fatigue during prolonged exercise in the heat. J Appl Physiol 1999;86:1032-9.

20 Schwid SR, Petrie MD, Murray R, et al. A randomized controlled study of the acute and chronic effects of cooling therapy for MS. Neurology 2003;60:1955-60.

21 Grahn DA, Murray JV, Heller HC. Cooling via one hand improves physical performance in heat-sensitive individuals with multiple sclerosis: a preliminary study. BMC Neurol 2008;8:14.

22 Op't Eijnde B, Keytsman C, Wens I, et al. Whole-body cooling does not compromise muscle oxidative capacity in subjects with multiple sclerosis. NeuroRehabilitation 2014;35:805-11.

23 Grover G, Ploughman M, Philpott DT, et al. Environmental temperature and exercise modality independently impact central and muscle fatigue among people with multiple sclerosis. Mult Scler J Exp Trans/ Clin 2017;3:205521731774762.

24 White AT, Wilson TE, Davis SL, et al. Effect of precooling on physical performance in multiple sclerosis. Mult Scler 2000;6:176-80.

25 Devasahayam AJ, Chaves AR, Lasisi WO, et al. Vigorous cool room treadmill training to improve walking ability in people with multiple sclerosis who use ambulatory assistive devices: a feasibility study. BMC Neurol 2020;20:33.

26 Leavitt VMet al. Aspirin is an effective pretreatment for exercise in multiple sclerosis: a double-blind randomized controlled pilot trial. Mult. Scler. J 2017.

27 Hersh EV, Moore PA, Ross GL. Over-the-counter analgesics and antipyretics: a critical assessment. Clin Ther 2000;22:500-48.

28 Wingerchuk DM, Benarroch EE, O'Brien PC, et al. A randomized controlled crossover trial of aspirin for fatigue in multiple sclerosis. Neurology 2005;64:1267-9.

29 Shaygannejad V, Janghorbani M, Ashtari F, et al. Comparison of the effect of aspirin and amantadine for the treatment of fatigue in multiple sclerosis: a randomized, blinded, crossover study. Neurol Res 2012;34:854-8.

30 Symmons DPM. Safety profile of low-dose aspirin. Lancet 1996;348:1394-5.

31 Thompson AJ, Banwell BL, Barkhof F, et al. Diagnosis of multiple sclerosis: 2017 revisions of the McDonald criteria. Lancet Neurology 2018;17:162-73.

32 Benedict RHB, Fishman I, McClellan MM, et al. Validity of the beck depression inventory-fast screen in multiple sclerosis. Mult Scler 2003;9:393-6.

33 Leavitt VM, Blanchard AR, Guo C-Y, et al. Aspirin is an effective pretreatment for exercise in multiple sclerosis: a double-blind randomized controlled pilot trial. Mult Scler 2018;24:1511-3.

34 Booth $\mathrm{G}$. Changes in temperature of the skin following the ingestion of food. Arch Intern Med 1936;57:533-43.

35 Chamberlain JM, Terndrup TE, Alexander DT, et al. Determination of normal ear temperature with an infrared emission detection thermometer. Ann Emerg Med 1995;25:15-20.

36 Henker R, Coyne C. Comparison of peripheral temperature measurements with core temperature. AACN Clin Issues 1995:6:21-30.

37 Bayer Inc. Consumer Care. Product Monograph Aspirin, 2017. Available: https://www.bayer.ca/omr/online/aspirin-pm-en.pd

38 Riebe D, Franklin BA, Thompson PD, et al. Updating ACSM's recommendations for exercise Preparticipation health screening. Med Sci Sports Exerc 2015;47:2473-9. 
39 American Thoracic Society; American College of Chest Physicians. ATS/ACCP statement on cardiopulmonary exercise testing. Am J Respir Crit Care Med Online Ser 2003:211-77.

40 Zigmond AS, Snaith RP. The hospital anxiety and depression scale. Acta Psychiatr Scand 1983;67:361-70.

41 Krupp LB. The fatigue severity scale: application to patients with multiple sclerosis and systemic lupus erythematosus. Arch. Neurol 1989;46:1121.

42 Washburn RA, Smith KW, Jette AM, et al. Washburn RA, Smith $\mathrm{KW}$, Jette am, Janney $\mathrm{Ca}$. The physical activity scale for the elderly (PASE): development and evaluation. J Clin Epidemiol 1993;46:153-62.

43 Borg GA. Psychophysical bases of perceived exertion. Med Sci Sports Exerc 1982;14:377-81.
44 Morrison EH, Cooper DM, White LJ, et al. Ratings of perceived exertion during aerobic exercise in multiple sclerosis. Arch Phys Med Rehabil 2008;89:1570-4.

45 Humphreys MA, Hancock M, Humphreys MA, et al. Do people like to feel 'neutral'?: Exploring the variation of the desired thermal sensation on the ASHRAE scale. Energy Build 2007;39:867-74.

46 Collett J, Meaney A, Howells K, et al. Acute recovery from exercise in people with multiple sclerosis: an exploratory study on the effect of exercise intensities. Disabil Rehabil 2017;39:551-8.

47 Wellek S, Blettner M. On the proper use of the crossover design in clinical trials. Dtsch Aerzteblatt Online 2012.

48 Dalgas U, Hvid LG, Kwakkel G, et al. Moving exercise research in multiple sclerosis forward (the MoXFo initiative): developing consensus statements for research. Mult Scler 2020;26:1303-8. 